\title{
Integrating Technology into Clinical Care to Improve Outcomes in Panic Disorder: Use of Safety Behaviors and Resulting Anxiety as Assessed by Smartphone-Based Experience Sampling Methods
}

Amanda W Baker ${ }^{1}$, PhD; Olivia M Losiewicz ${ }^{1}$, AB; Samantha N Hellberg ${ }^{1}$, BA; Naomi M Simon ${ }^{1,2}$, PhD

${ }^{1}$ Massachusetts General Hospital, Boston, MA, United States

${ }^{2}$ New York University, New York, NY, United States

Corresponding Author:

Amanda W Baker, PhD

Massachusetts General Hospital

1 Bowdoin Square

Boston, MA,

United States

Phone: 7266944

Email: awbaker1@partners.org

\begin{abstract}
Background: Research in mental health conditions such as panic disorder suffers from issues related to the means of assessing the condition and its contributing factors by self-report in the office setting. The integration of real time technologies into research is critically needed. Panic disorder is the fifth leading cause of missed work days across all chronic medical conditions, and anxiety disorders are estimated to cost more than $\$ 40$ billion annually in the United States (Greenberg et al, 1999). Panic attacks occur in approximately $23 \%$ of the general population (Kessler et al, 2006) and cause substantial functional and social impairment, as well as significant financial burden on the health care system. While effective treatments for panic disorder exist, more than half of patients do not improve, remain symptomatic post-treatment, or return to treatment within two years (Brown \& Barlow, 1995; Gloster et al, 2013). Very little is known about what treatment will work for each patient and why. New technologies allow us to identify individual factors that may be important to treatment outcomes. This pilot study aimed to bridge the gap between the human element and technology using smartphones to more efficiently investigate a factor that may contribute to lack of remission in panic disorder, the use of safety behaviors. Safety behaviors represent ineffective attempts to reduce or eliminate anxiety (eg, carrying a water bottle to reduce physiological sensations that arise during anxiety; Helbig-Lang \& Petermann, 2010). They are hypothesized with mixed evidence to play a central role in the etiology and maintenance of anxiety disorders, including panic disorder. Existing studies are limited in their temporal conclusions and ecological validity. New technologies such as smartphones permit time-intensive investigation of these phenomena in the natural environment in which they occur, thus improving external validity.
\end{abstract}

Objective: To examine the effect of safety behavior use on anxiety response in panic disorder using smartphone-based ecological momentary assessment.

Methods: Participants (N=13) were adults with panic disorder. For 14 days, participants answered a brief smartphone-based questionnaire of panic symptom severity and safety behavior use 5 times a day.

Results: Analyses were conducted using $\mathrm{N}=910$ data points from participants ( $\mathrm{N}=13$ ). Safety behavior use was highly correlated with anxiety and predictive of later anxiety level. Increased safety behavior use at time 1 predicted increased anxiety at times 2 , 3,4 , and $5(\mathrm{t}[1,100]$ values $>4.26$; $\mathrm{P}$ values $<.001)$. Safety behavior use at time 1 was a significant predictor of anxiety at time 2 , even when controlling for anxiety at time $1(\mathrm{t}[2,103]=2.83 ; P=.006)$.

Conclusions: This study was novel in its approach to combine smartphone-based ecological momentary assessment with traditional clinical report, overcoming challenges in later retrospective reporting such as temporality and recall biases. In line with theoretical conceptualizations of panic disorder, our findings support that individuals engage in safety behaviors when anxious and that safety behavior use then robustly maintains and even heightens anxiety. Future directions for novel technological, statistical, and personalized approaches to expand our understanding of safety behaviors in anxiety disorders and implications for treatment will be discussed. 
(iproc 2018;4(2):e11788) doi: $10.2196 / 11788$

\section{KEYWORDS}

anxiety; anxiety disorders; ecological momentary assessment; panic disorder; smartphone; treatment

Edited by T Hale; this is a non-peer-reviewed article. Submitted 02.08.18; accepted 29.08.18; published 17.09.18.

Please cite as:

Baker AW, Losiewicz OM, Hellberg SN, Simon NM

Integrating Technology into Clinical Care to Improve Outcomes in Panic Disorder: Use of Safety Behaviors and Resulting Anxiety

as Assessed by Smartphone-Based Experience Sampling Methods

iproc 2018;4(2):e11788

URL: http://www.iproc.org/2018/2/e11788/

doi: $\underline{10.2196 / 11788}$

PMID:

(C)Amanda W Baker, Olivia M Losiewicz, Samantha N Hellberg, Naomi M Simon. Originally published in Iproceedings (http://www.iproc.org), 17.09.2018. This is an open-access article distributed under the terms of the Creative Commons Attribution License (https://creativecommons.org/licenses/by/4.0/), which permits unrestricted use, distribution, and reproduction in any medium, provided the original work, first published in Iproceedings, is properly cited. The complete bibliographic information, a link to the original publication on http://www.iproc.org/, as well as this copyright and license information must be included. 